\title{
Digitaler Kodex
}

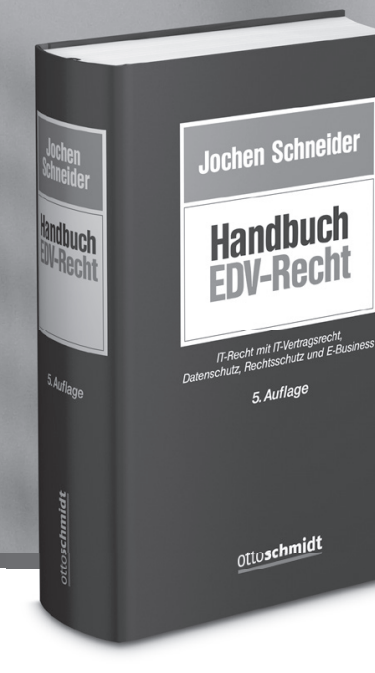

Jochen Schneider

\section{Handbuch EDV-Recht}

IT-Recht mit IT-Vertragsrecht, Datenschutz, Rechtsschutz und E-Business. Herausgegeben von RA Prof. Dr. Jochen Schneider. Bearbeitet von RA Ludwig Antoine, RA Frieder Backu, RAin Dr Irene Bayer, RAin Elke Bischof, RAin Isabell Conrad, RA Thomas Graf, LL.M., RAin Ines Hassemer, RAin Danielle Hertneck, RA Fabian Kahlert, RA Prof. Dr. Timoleon Kosmides, RAin Dr. Romina Polley, RA Prof. Dr. Jochen Schneider, RA Bernd Suchom ski, LL.M., und RAin Michaela Witzel, LL.M. 5., neu bearbeitete Auflage 2017, 3.312 Seiten, Lexikonformat, gbd. 199,- €. ISBN 978-3-504-56094-2
Der Begriff „digitale Revolution“ fällt seit einigen Jahren, um zu beschreiben, wie umfassend der informationstechnologische Fortschritt die industrielle Produktion, die Kommunikation und den Handel umwälzt und damit zugleich das IT- und Datenschutzrecht vor neue Herausforderungen stellt.

Das nun in fünfter Auflage erschienene „Handbuch EDV-Recht“ ist der digitale Kodex für das Zeitalter der Informationstechnologie. Professor Dr. Jochen Schneider begleitet, erschließt und prägt das IT- und Datenschutzrecht seit seiner Entstehung.

Die zahlreichen Mustertexte, Beispiele und Verweise auf maßgebliche Rechtsprechung und Literatur machen das Werk zu einem Muss für alle IT-Rechtspraktiker.

Leseprobe und Bestellung unter www.otto-schmidt.de/sc5 


\section{Bestens versorgt bei der \\ Vertragsgestaltung im IT-Recht}

\section{Handbuch der IT-Verträge}

Herausgegeben von RA, FA IT-Recht, Dipl.-Informatiker Dr. Helmut Redeker. Loseblatt, 3 Bände. Grundwerk mit Fortsetzungsbezug für mindestens 2 Jahre nur 159,- €, 3 Ergänzungslieferungen pro Jahr.

ISBN 978-3-504-56008-9.

Das Werk online: juris.de/pmitr

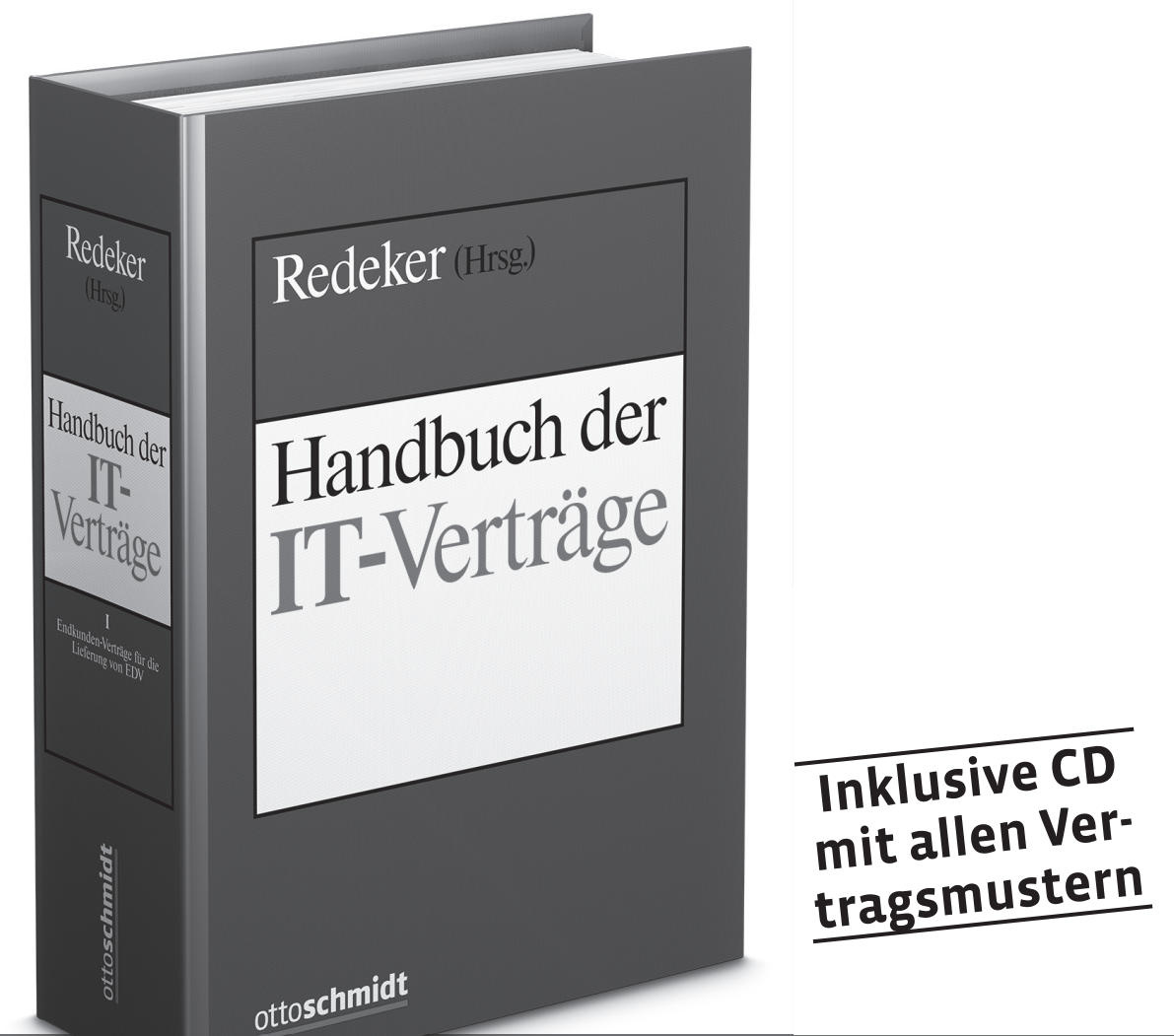

Das praxisnahe Werk zur Vertragsgestaltung versorgt Rechtsberater und Entscheider in Unternehmen in verschiedensten IT- und telekommunikationsrechtlichen Bereichen mit ausführlich kommentierten Vertragsmustern. Klausel für Klausel nehmen erfahrene Praktiker zu allen praxisrelevanten Fragen u.a. des IT-Vertragsrechts, des Internetrechts und des Telekommunikationsrechts Stellung. Sonderfälle werden mit Alternativklauseln und -mustern berücksichtigt.

Aktualisiert in der Mai-Lieferung 2018:

- Hardware-Miete/System-Miete

- Handelsvertretervertrag Hardware

- Webdesign-Vertrag

- Auftragsverarbeitung

Ausführliche Informationen, Bestellung und Leseprobe unter www.otto-schmidt.de/riv 


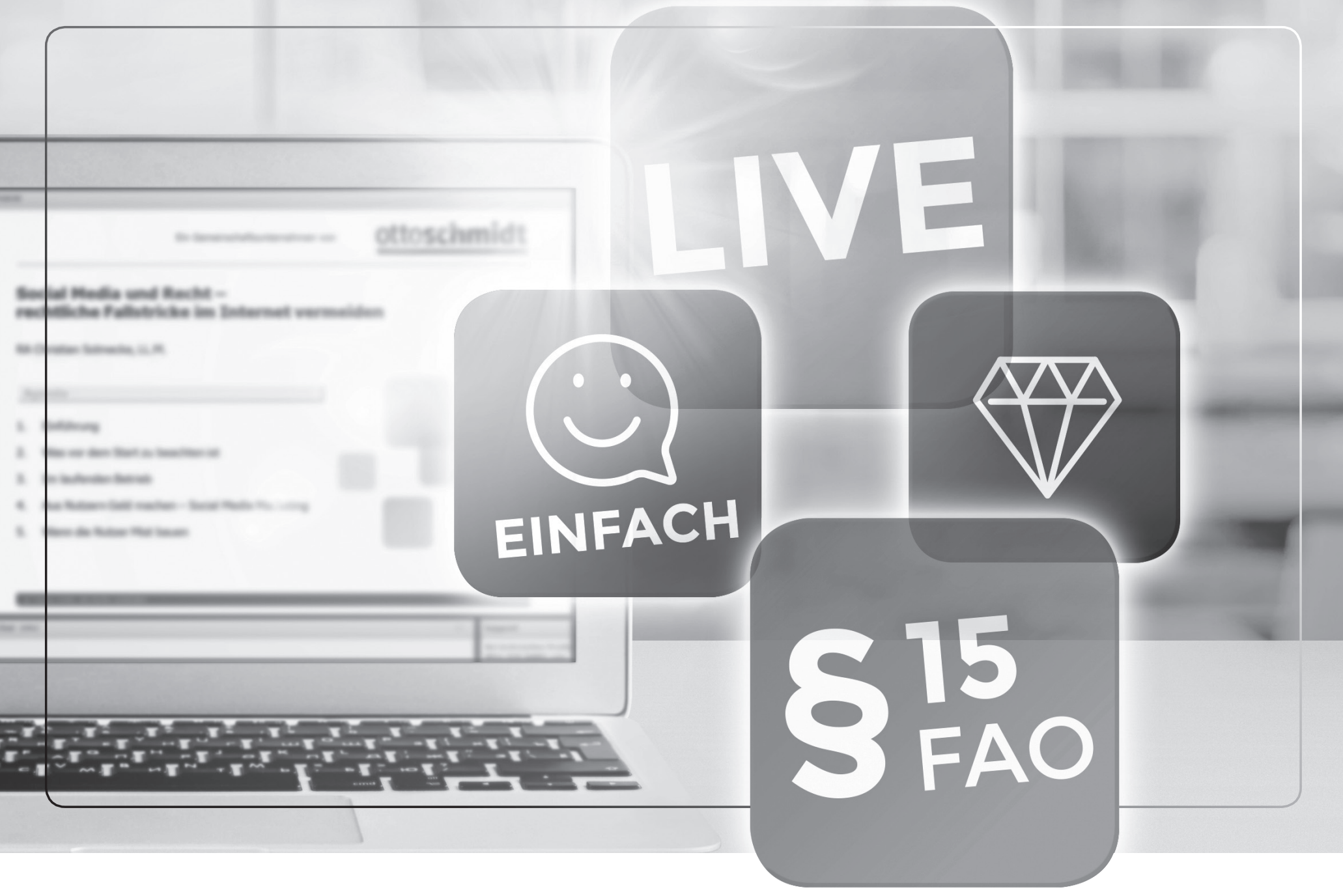

\section{Fortbildung für Rechtsanwälte: die neuen Online-Live-Seminare}

\section{Aktuelle Terminauswahl:}

Neue Datenschutzbestimmungen seit 25.05.2018 - Eine Anleitung zur Umsetzung in Ihrer Kanzlei

19.06.2018 | 18.09.2018

RA Dr. Astrid Auer-Reinsdorff
Weil es so einfach ist: anmelden, einloggen, fortbilden! Absolvieren Sie die neuen Online-Live-Seminare von Otto Schmidt ganz bequem in Ihrem Büro oder zu Hause. In Kooperation mit dem TeleLex-Angebot der DATEV bieten wir Ihnen alle Möglichkeiten moderner und zeitsparender Fortbildung.

- Kompetent \& persönlich: Top-Dozenten und Fragen via Live-Chat.

- Einfach \& flexibel: Mit nur wenigen Mausklicks noch bis zu zwei Stunden vor Seminarbeginn anmelden.

- Günstig \& effizient: Für nur 95 Euro - inklusive Zertifikat, ganz ohne Reisekosten.

Jetzt informieren und buchen: alle Themen und Termine unter otto-schmidt.de/telelex 


\section{juris PartnerModul Medienrecht}

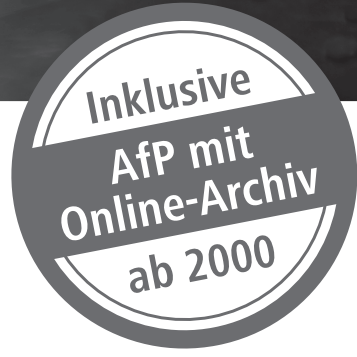

partnered by C.F. Müller | De Gruyter | dfv Mediengruppe | Verlag Dr. Otto Schmidt | Verlag Medien und Recht

Das juris PartnerModul Medienrecht unterstützt Sie in allen Fragen des nationalen und europäischen Telekommunikations- und Rundfunkrechts und stellt dabei den Bezug zu Verlags-, Leistungsschutz- und Wettbewerbsrecht sowie sämtlichen angrenzenden Rechtsgebieten her.

Im Mittelpunkt steht die Kommentierung des nationalen und europäischen Medienrechts: die Durchsetzung des geistigen Eigentums, das Wettbewerbs- und Werberecht, der Schutz von Medienprodukten, Persönlichkeitsrecht und Medienstrafrecht sowie IT-Recht.

Alle wichtigen Rechtsvorschriften und Vereinbarungen, vom Verfassungsrecht über die Regelungen mit den Rundfunkanstalten zu Rundfunkgebühren bis zum Jugend- und Datenschutz, sind dokumentiert. Die enthaltenen Archive von Fachzeitschriften wie AfP, K\&R und MR/MR-int garantieren absolute Aktualität. Alle Werke sind für die OnlineNutzung in der bewährten juris Qualität aufbereitet.

Inhalt

- Das Recht der Anzeige, Rath-Glawatz/Engels/Dietrich

- Das Recht der Wort- und Bildberichterstattung, Wenzel

- juris PraxisKommentar Internetrecht, Heckmann (Hrsg.)

- Kommunikation \& Recht, K\&R

- Medienrecht, Ring

- Medienrecht Praxishandbuch, Wandtke/Ohst

- Presserecht, Soehring/Hoene

- Pressevertriebsrecht, Mann/Smid

- Rundfunkstaatsvertrag Jugendmedienschutz-Staatsvertrag, Hartstein/Ring u. a.

- Zeitschrift für das gesamte Medienrecht, AfP

- und viele weitere Titel

$\oplus$ Rechtsprechung, Gesetze und Literaturnachweise von juris

Mehr Informationen unter: www.juris.de/medienrecht
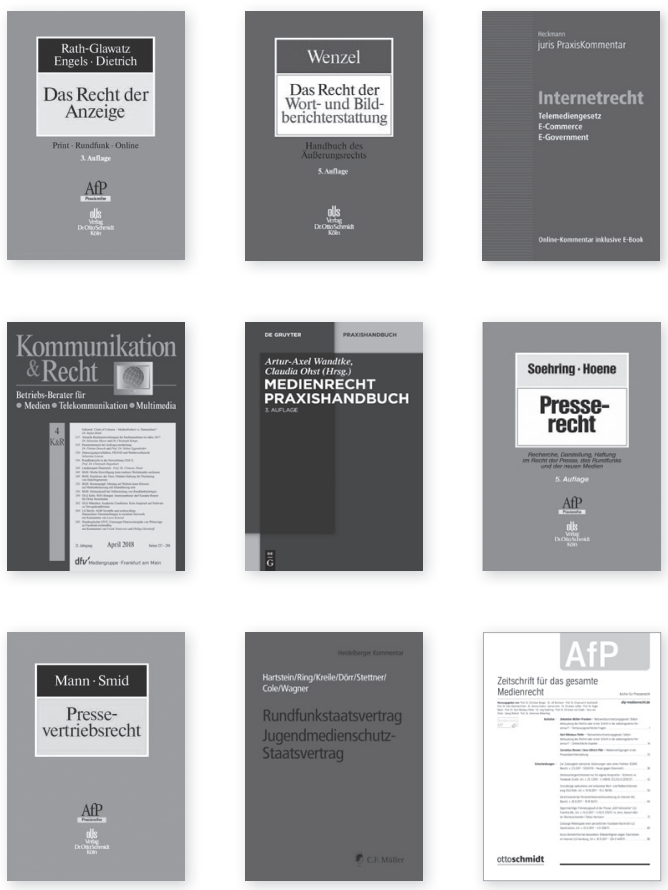


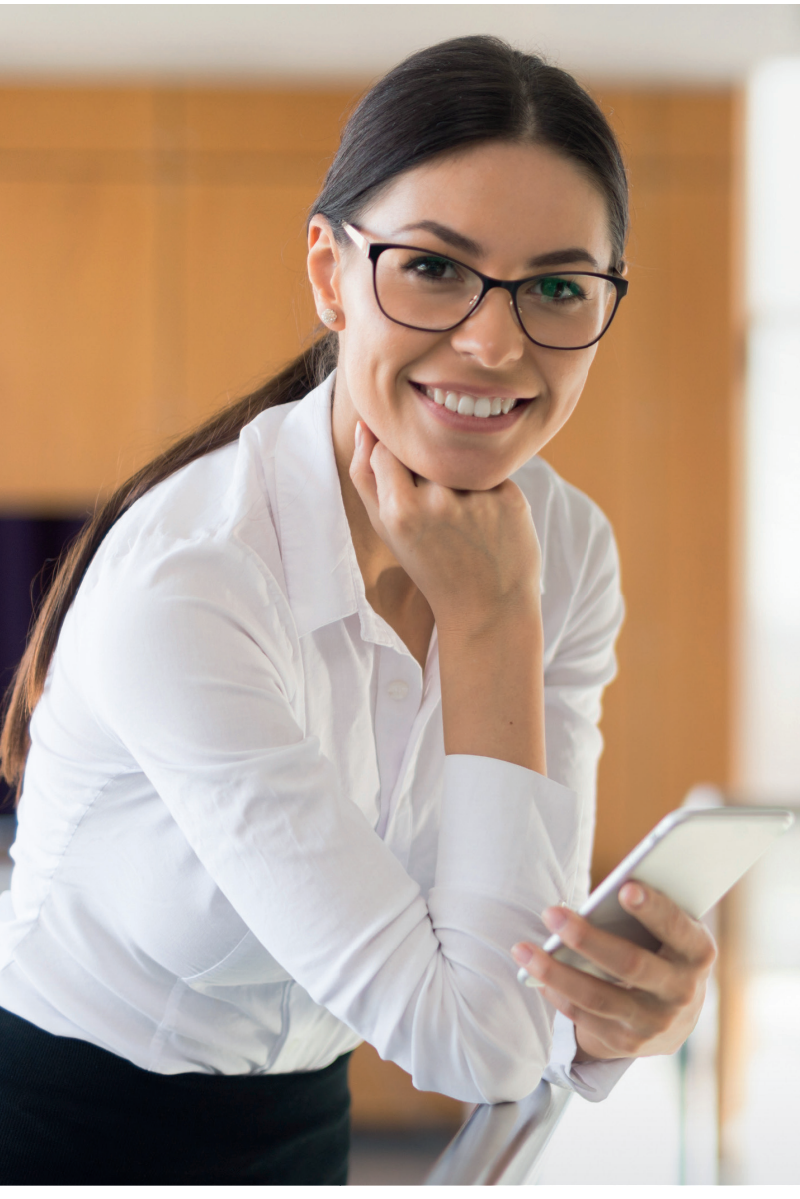

\section{JETZT 4 WOCHEN GRATIS NUTZEN!}

\section{Aktionsmodul Zivilrecht}

\section{Zöller Zivilprozessrecht}

\section{Zivil- und Zivilverfahrensrecht}

\section{Arbeitsrecht}

\section{> Familienrecht}

\section{> Miet- und WEG-Recht}

Ihre Online-Bibliothek mit mehr als 20 Prozent Preisvorteil

\section{Module, 3 Nutzer, 1 Preis:}

nur 59 EUR mtl./zzgl. MwSt statt 79,90 EUR

Bewährte Kompetenz in zukunftsweisendem Format! Die Datenbank von Otto Schmidt sorgt für mehr Aktualität und Komfort in Ihrem Arbeitsalltag:

• Führende Kommentare, Handbücher und Zeitschriften

- Meinungsbildend, umfassend und tiefgehend

- Rechtssicherheit und Zitierfähigkeit

- Gesetze und Entscheidungen im Volltext

- Inklusive Selbststudium mit Zertifikat nach § 15 FAO

Jetzt 4 Wochen gratis nutzen: otto-schmidt.de/akr

\section{ottoschmidt}




\section{Äußerst recht im Äußerungsrecht!}

Wenzel

Das Recht der Wort- und Bildberichterstattung Handbuch des Äußerungsrechts

Begründet von RA Prof. Dr. Karl Egbert Wenzel t. Fortgeführt von RA Dr. Emanuel H. Burkhardt; RA Dr. Waldemar Gamer t; Prof. Dr. Karl-Nikolaus Peifer; RA Prof. Joachim Ritter von Strobl-Albeg. 6. neu bearbeitete Auflage 2018, ca. 1200 Seiten, Lexikonformat, gbd. 179,- $€$. ISBN 978-3-504-15675-6

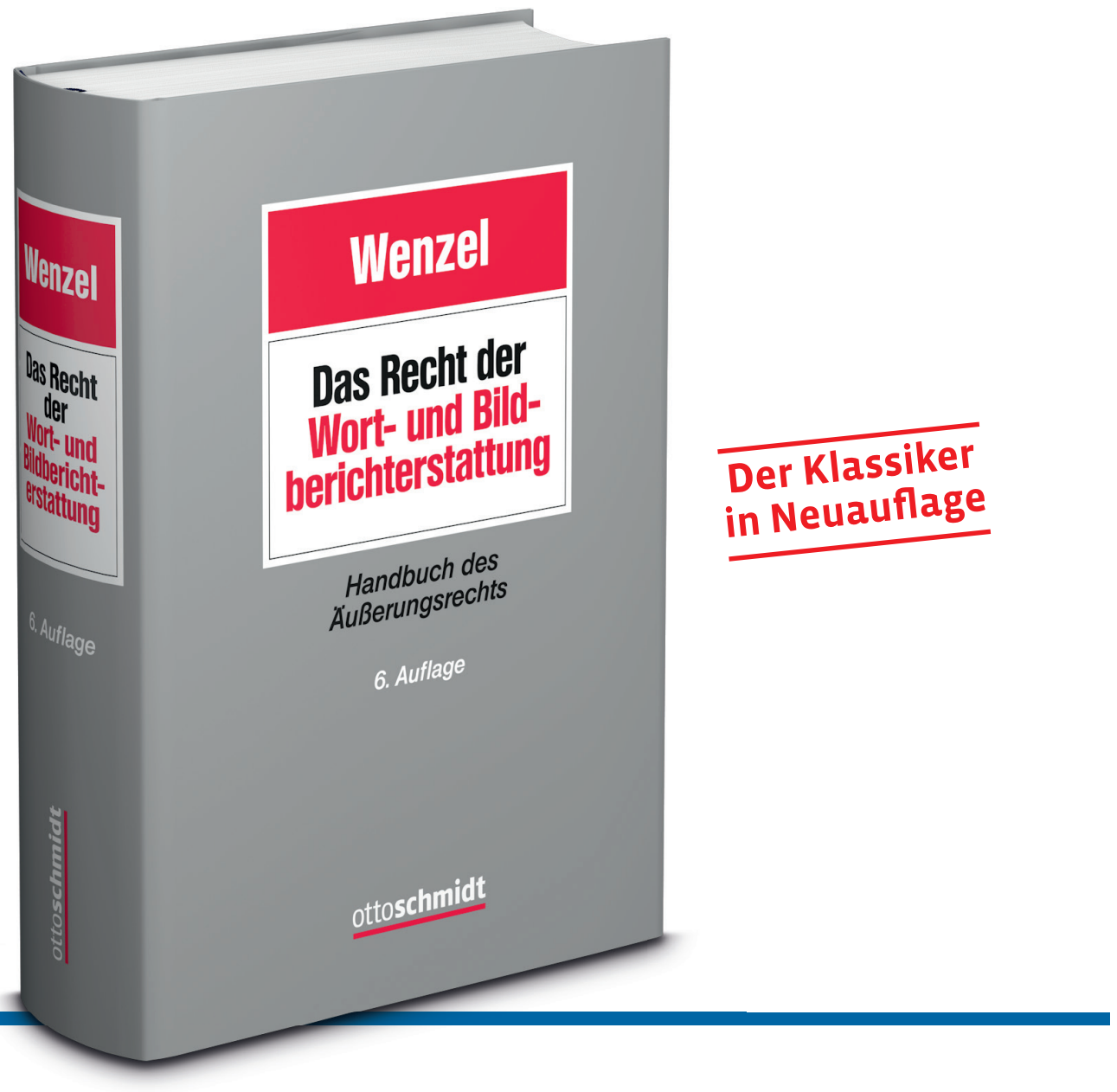

Das unentbehrliche Standardwerk für Jeden, der mit dem Äußerungsrecht zu tun hat, ist wieder auf dem neuesten Stand. Was man veröffentlichen darf und was nicht: Im Wenzel steht es drin. Anschaulich und praxisnah. Die gesetzlichen Neuerungen wie zuletzt das Netzwerkdurchsetzungsgesetz und die umfangreiche Rechtsprechung wurden akribisch ausgewertet und in die Neuauflage eingearbeitet.

Die gelungene systematische Darstellung der gesamten Materie weist den Weg durch dieses stark von Kasuistik geprägte Gebiet. Damit wird der schnelle Zugang zu Lösungen im Äußerungsrecht ermöglicht sowie Antworten für konkrete Fragestellungen gegeben. Der Wenzel ist ihr verlässlicher Partner bei allen Rechtsfragen im Spannungsfeld zwischen Meinungsfreiheit und Persönlichkeitsschutz.

Überzeugen Sie sich bei einer Leseprobe unter www.otto-schmidt.de/wz6 